\title{
Horseshoes, Homoclinic Connections and Global Chaos in Current-Mode Controlled DC/DC Converters
}

\author{
Dong Dai ${ }^{\dagger}$, Yue $\mathrm{Ma}^{\ddagger}$ and Chi K. Tse ${ }^{\dagger}$ \\ ${ }^{\dagger}$ Department of Electronic and Information Engineering, Hong Kong Polytechnic University, Hong Kong \\ ${ }^{\ddagger}$ Department of Electrical and Electronic Engineering, Tokushima University, Japan
}

\begin{abstract}
In this paper, chaos in a current-mode controlled boost converter is studied. Firstly, the existence of chaos is proven theoretically in this system. The proof consists of showing that the dynamics of the system is semiconjugate to that of a shift map, which implies positive entropy of the system and hence chaotic behavior. The essential tool is the horseshoe hypotheses proposed by Kennedy and Yorke, which will be reviewed prior to the discussion of the main finding. Then, the existence of chaos is illustrated in the light of homoclinic connection. Furthermore, global chaos resulting from homoclinic intersection of stable and unstable manifolds are illustrated numerically.
\end{abstract}

\section{INTRODUCTION}

Switching power converters have been shown to exhibit a variety of complex behavior such as bifurcation and chaos [1]-[3]. Although chaotic behavior has been widely recognized in switching power converters, no rigorous proof of chaos in such systems has been reported. Also, the mechanism of chaos in such systems has rarely been investigated. Up to now, only a discussion of the existence of a Smale horseshoe mechanism in a voltage-mode controlled buck converter was reported by Olivar et al. [4]. The main purpose of this paper is to provide a rigorous proof of chaos in a current-mode controlled boost converter. This is done by proving the existence of a horseshoe in the describing Poincare map. The proof is based on the work of Kennedy and Yorke [5], which gives a set of sufficient conditions for a continuous map to exhibit chaotic behavior. Then, we illustrate the existence of chaos by observing homoclinic connections of the stable and unstable manifolds. Furthermore, we report homoclinic tangency and global chaos in the current-mode controlled boost converter.

\section{THE System}

The system studied here is a current-mode controlled boost converter shown in Fig. 1, which has been found to be chaos prone by simulation and experimental studies [6], [7]. The state variables for this system are the inductor current, $i$, and the output voltage, $v$. For simplicity, we consider continuous-conduction-mode (CCM) operation, in which the inductor current never falls to zero. The Poincaré map for this system is

$$
x_{\mathrm{n}+1}=f\left(x_{n}, \mu\right)
$$

where $x_{n}$ is a vector consisting of the state variables at $t=n T, T$ being the switching period, i.e.,

$$
x_{n}=\left(\begin{array}{c}
i_{n} \\
v_{n}
\end{array}\right)=\left(\begin{array}{c}
i(n T) \\
v(n T)
\end{array}\right) .
$$

Typically, the inductor current waveform is shown in Fig. 2. Clearly, depending on the value of $i$ at the beginning of the period, the switch may or may not turn off during a switching period.

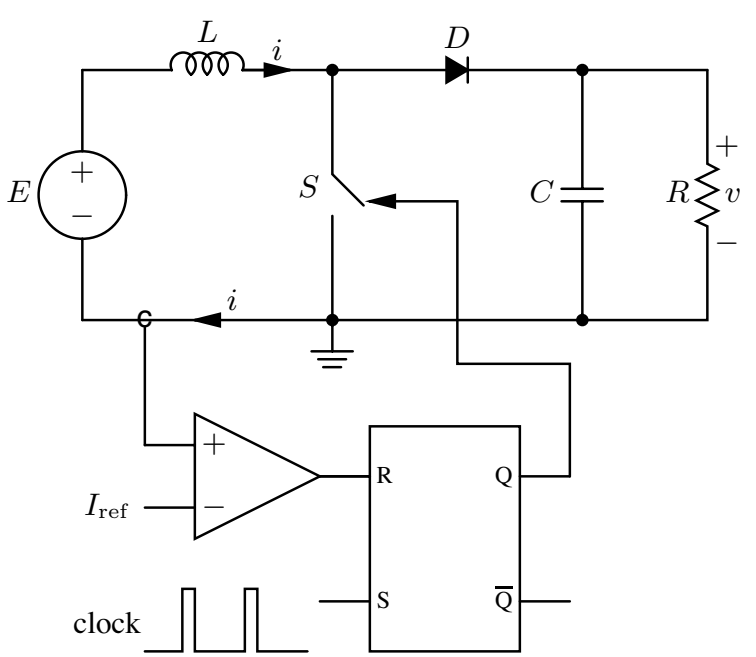

Fig. 1. Current-mode controlled boost converter.

Specifically, provided $I_{\mathrm{ref}}-i_{n}<E T / L$, the operation is as described above, with the switch turned off once during a period, as illustrated in Fig. 2 (b). However, if $I_{\text {ref }}-i_{n} \geq E T / L$, the switch remains on for the whole period, as shown in Fig. 2 (a). Thus, the corresponding Poincaré map can be written as [6]:

$$
\left(\begin{array}{c}
i_{n+1} \\
v_{n+1}
\end{array}\right)=\left(\begin{array}{c}
i_{n}+\frac{E T}{k_{n}} \\
v_{n} e^{-2 T}
\end{array}\right)
$$

for the case $I_{\text {ref }}-i_{n} \geq \frac{E T}{L}$, but is given by ${ }^{1}$

$$
\left(\begin{array}{c}
i_{n+1} \\
v_{n+1}
\end{array}\right)=\left(\begin{array}{c}
e^{-k \tau}\left(A_{1} \sin (\omega \tau)+A_{2} \cos (\omega \tau)\right)+\frac{E}{R} \\
E-\frac{e^{-k \tau}}{\omega}\left[\left(k v_{n}^{\prime}-k E-\frac{A_{2}}{C}\right) \sin (\omega \tau)\right. \\
\left.+\left(E-v_{n}^{\prime}\right) \cos (\omega \tau)\right]
\end{array}\right)
$$

for the case $I_{\text {ref }}-i_{n}<\frac{E T}{L}$, where $k=\frac{1}{2 R C}, \omega=\sqrt{\frac{1}{L C}-k^{2}}$, $\tau=T-\frac{L}{E}\left(I_{\text {ref }}-i_{n}\right), v_{n}^{\prime}=v_{n} e^{-2 k(T-\tau)}, A_{2}=I_{\text {ref }}-\frac{E}{R}$ and $A_{1}=\frac{k L A_{2}+E-v_{n}^{\prime}}{\omega L}$. Finally, as an operational requirement for CCM, we need to ensure a sufficiently large inductance or short period.

\section{REVIEW OF THE HORSESHOE HyPOTHESES}

We begin with a review of some aspects of the topological horseshoe theory proposed by Kennedy and Yorke [5], which are relevant to the main result to be presented in this paper. Essentially, Kennedy and Yorke found a set of sufficient conditions for a continuous map $f$

${ }^{1}$ The Poincare map is a piecewise smooth map and is noninvertible as the injective condition may not be satisfied from Fig. 2 . 


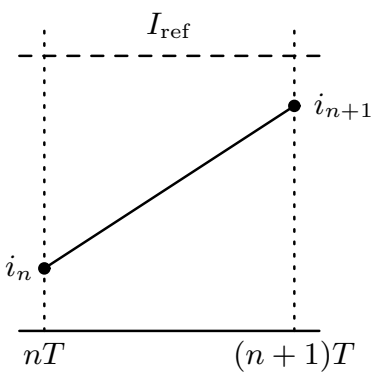

(a)

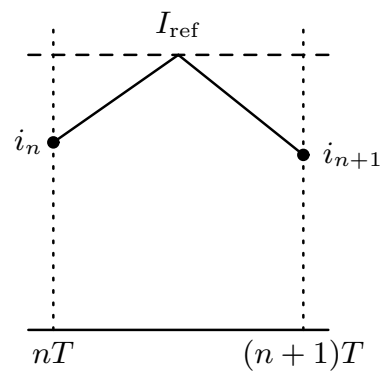

(b)
Fig. 2. Typical inductor current waveforms (a) for $I_{\text {ref }}-i_{n} \geq E T / L$; and (b) for $I_{\mathrm{ref}}-i_{n}<E T / L$.

to exhibit chaos. These conditions are summarized in what has been called the horseshoe hypotheses, which include the following:

1) $X$ is a separable metric space;

2) $Q \subset X$ is locally connected and compact;

3) The map $f: Q \rightarrow X$ is continuous;

4) The sets end f $_{0} \subset Q$ and end ${ }_{1} \subset Q$ are disjoint and compact, and each component of $Q$ intersects both end $\mathbf{d}_{0}$ and end $\mathbf{e}_{1}$;

5) $Q$ has crossing number $N \geq 2$.

The crossing number $N$ in the above hypotheses can be reasoned as follows. A connection $\Gamma$ is a compact connected subset of $Q$ that intersects both sets end $\mathbf{d}_{0}$ and end $\mathbf{d}_{1}$. A preconnection $\gamma$ is a compact connected subset of $Q$ for which $f(\gamma)$ is a connection. Then, the crossing number $N$ is defined as the largest number such that every connection contains at least $N$ mutually disjoint preconnections.

Kennedy-Yorke's Theorem [5] Consider a map $f$. Suppose the horseshoe hypotheses are satisfied. Then, there is a closed invariant set $\Lambda \subset Q$ for which $f \mid \Lambda$ is semiconjugate to a one-sided shift map on $N$ symbols. ${ }^{2}$

The main consequence of this result is that the entropy of $f$ is not less than that of $\sigma$, which implies that $f$ has positive entropy and is therefore chaotic.

\section{Horseshoe in CurRent-Mode Controlled Boost CONVERTER}

In this section, the existence of a horseshoe in a specific boost converter will be proven theoretically by using Kennedy-Yorke's theorem. The parameters used here are: $T=100 \mu \mathrm{s}, R=20 \Omega$, $L=1.5 \mathrm{mH}, C=12 \mu \mathrm{F}, E=10 \mathrm{~V}$ and $I_{\text {ref }}=3.5 \mathrm{~A}$.

Denote the afore-described Poincaré map by $p$. Here, we consider two iterates of $p$ and denote it by $P$, i.e., $P=p^{2}{ }^{3}$ Consider the parallelogram $A B C D$ with the coordinates of four endpoints defined as $A=(3,18), B=(3.46,18.8), C=(2.88,29)$ and $D=$ $(3.34,29.8)$. Figure 3 shows the parallelogram $A B C D$ and its image $A^{\prime} B^{\prime} C^{\prime} D^{\prime}$ under $P$. Here, $A^{\prime}=P(A), B^{\prime}=P(B), C^{\prime}=P(C)$ and $D^{\prime}=P(D)$. The image $A^{\prime} B^{\prime} C^{\prime} D^{\prime}$ is divided into two parts by the curve $E^{\prime} F^{\prime}$, i.e., $A^{\prime} B^{\prime} C^{\prime} D^{\prime}=A^{\prime} E^{\prime} F^{\prime} D^{\prime} \cup B^{\prime} E^{\prime} F^{\prime} C^{\prime}$, where $A^{\prime} E^{\prime} F^{\prime} D^{\prime}=P(A E F D), B^{\prime} E^{\prime} F^{\prime} C^{\prime}=P(B E F C)$ and $E^{\prime} F^{\prime}=$ $P(E F)$. It is noted that $A^{\prime} E^{\prime} F^{\prime} D^{\prime}$ intersects with $B^{\prime} E^{\prime} F^{\prime} C^{\prime}$ and $E^{\prime} F^{\prime} \subset O^{\prime} E^{\prime} F^{\prime}=A^{\prime} E^{\prime} F^{\prime} D^{\prime} \cap B^{\prime} E^{\prime} F^{\prime} C^{\prime}$.

Now, we choose the line $A D$ and $B C$ as the two end subsets required in the horseshoe hypotheses. To ease the construction of

\footnotetext{
${ }^{2}$ Suppose $\sigma$ is the shift map on two symbols. A shift map on $N$ symbols is denoted by $\sigma \mid \Sigma^{N}$. Also, if $f \mid \Lambda$ is semiconjugate to $\sigma \mid \Sigma^{N}$, there exists a continuous and onto map $\phi: \Lambda \rightarrow \Sigma^{N}$ such that $\phi \circ f=\sigma \circ \phi$. For details of the shift map, see [8].

${ }^{3}$ The map $P$ can also be regarded as the Poincare map with stroboscopic sampling period $2 T$ which is two times of that of $p$.
}

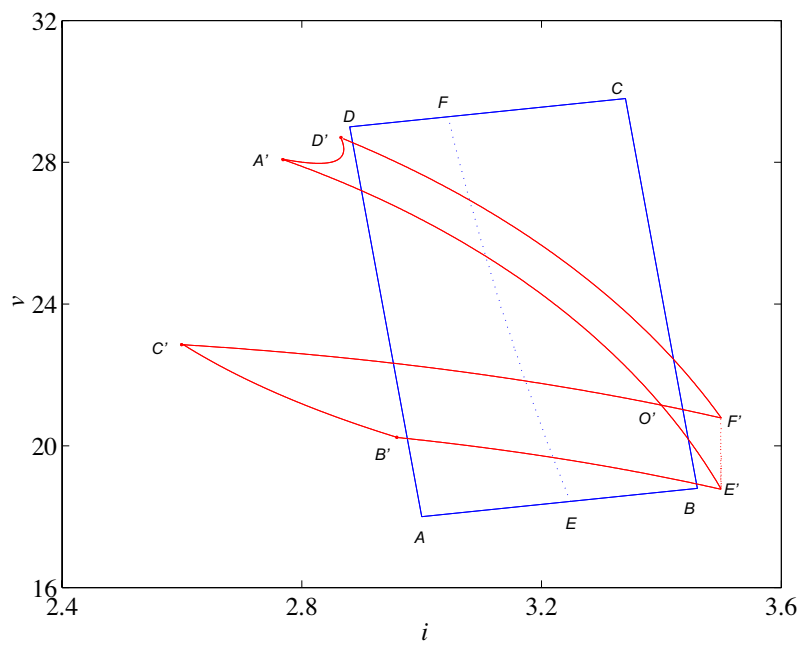

Fig. 3. $A B C D$ and its image $A^{\prime} B^{\prime} C^{\prime} D^{\prime}$ under $P$.

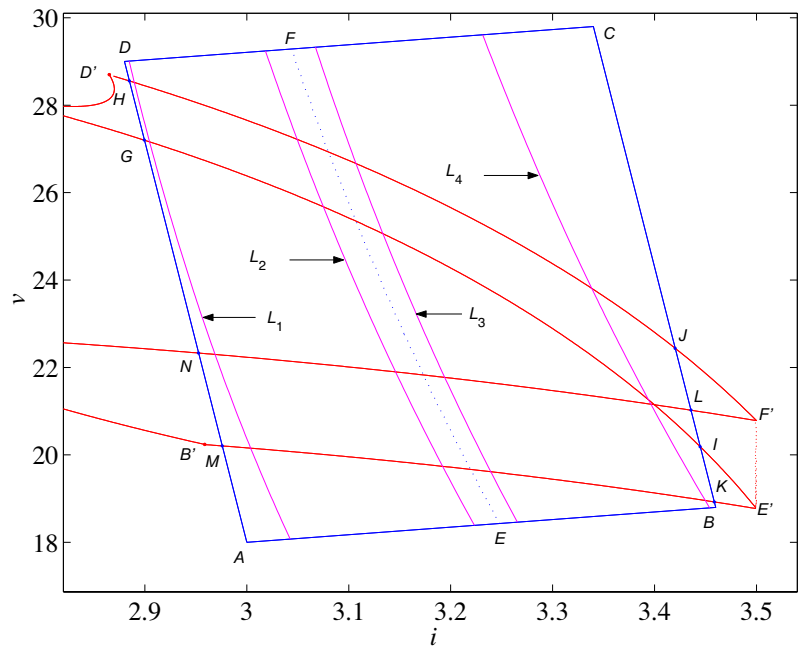

Fig. 4. Intersections of $A^{\prime} B^{\prime} C^{\prime} D^{\prime}$ with two ends ( $A D$ and $B C$ ) and preimages corresponding to these intersections.

the preconnections, we look at $A B C D$ and its image $A^{\prime} B^{\prime} C^{\prime} D^{\prime}$ again. It is straightforward to find that $A^{\prime} E^{\prime} F^{\prime} D^{\prime} \cap A D=G H$ and $A^{\prime} E^{\prime} F^{\prime} D^{\prime} \cap B C=I J$. Similarly, $B^{\prime} E^{\prime} F^{\prime} C^{\prime} \cap B C=K L$ and $B^{\prime} E^{\prime} F^{\prime} C^{\prime} \cap A D=M N$. By numerical computation, the corresponding preimages of the lines $G H, I J, K L$ and $M N$ can also be obtained, i.e., $P\left(L_{1}\right)=G H, P\left(L_{2}\right)=I J, P\left(L_{3}\right)=K L$ and $P\left(L_{4}\right)=M N$, as shown in Fig. 4. It should be noted that, in Fig. 4, the zone encompassed by $L_{1}$ and $L_{2}$ within $A B C D$ (denoted by $\Omega_{1}$ ) is disjoint to that by $L_{3}$ and $L_{4}$ (denoted by $\Omega_{2}$ ). It can also be concluded that any curve across the zone $\Omega_{1}\left(\Omega_{2}\right)$ with two ends located on $L_{1}\left(L_{3}\right)$ and $L_{2}\left(L_{4}\right)$ will be a preconnection. This will be useful for constructing the proof of the following theorem, which summarizes our main finding:

Main Theorem For the Poincaré map $P$ describing the dynamics of the current-mode controlled boost converter, there exists a closed invariant set $\Lambda \subset Q$, where $Q \subset \mathcal{R}^{2}$, such that $P \mid \Lambda$ is semiconjugate to a one-sided shift map.

Proof: Recall the horseshoe hypotheses. First, $\mathcal{R}^{2}$ (which is $X$ in the horseshoe hypotheses) is separable. Consider $Q=A B C D$ in the afore-described construction. Clearly, $Q \subset X$ is locally connected 


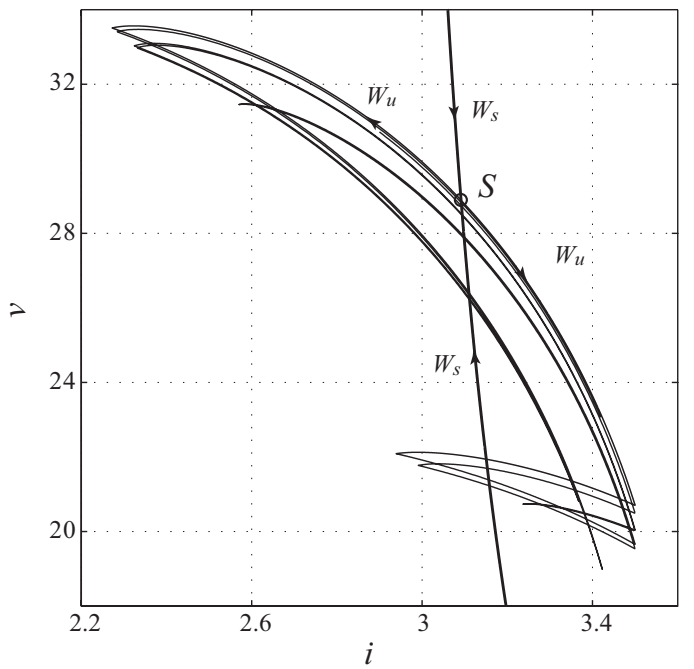

(a)

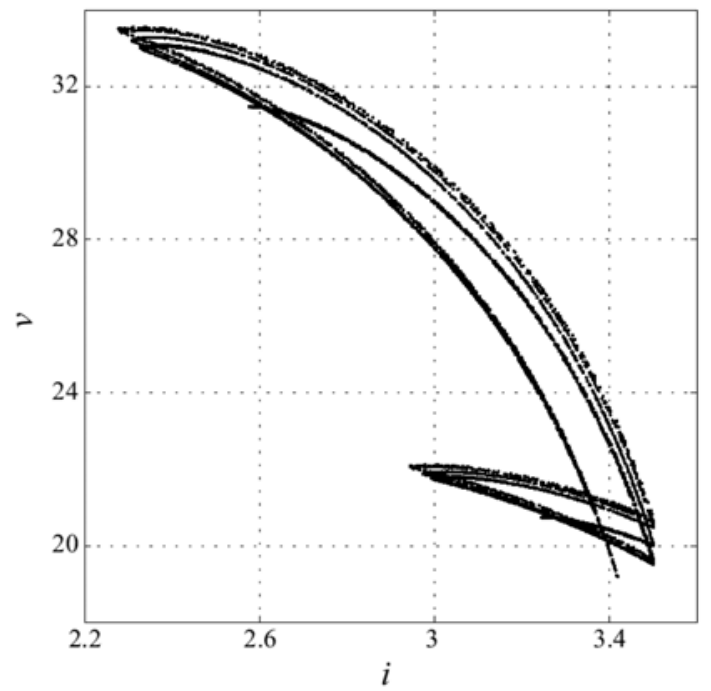

(b)

Fig. 5. (a) Stable $\left(W_{s}\right)$ and unstable $\left(W_{u}\right)$ manifolds of the saddle point $S$; (b) corresponding chaotic attractor.

and compact. The Poincaré map $P: Q \rightarrow X$ is continuous. Let end $_{0}=A D$ and end $\mathbf{d}_{1}=B C$. Then, they are disjoint and compact. The crossing number $N$ can be derived as follows. Referring to the afore-presented construction, given any connection $\Gamma$, it will cross both $\Omega_{1}$ and $\Omega_{2}$. Moreover, $\Omega_{1} \cap \Gamma$ and $\Omega_{2} \cap \Gamma$ are two mutually disjoint preconnections according to the previous statement. Thus, the crossing number $N \geq 2$. Hence, the horseshoe hypotheses are satisfied. From Kennedy-Yorke's theorem, there exists a closed invariant set $\Lambda \subset A B C D$ such that $P \mid \Lambda$ is semiconjugate to a onesided shift map.

\section{Homoclinic Connection and Global ChaOs}

In this section we examine the chaotic dynamics of the system from a different viewpoint, which provides indirect evidence of chaos in the system. Specifically, stable and unstable manifolds of the same fixed point of a map can intersect each other, which has been termed homoclinic intersection in much of the literature [9]. Smale [10] has shown that existence of homoclinic intersection implies horseshoetype dynamics for some sufficiently large number of iterations of the

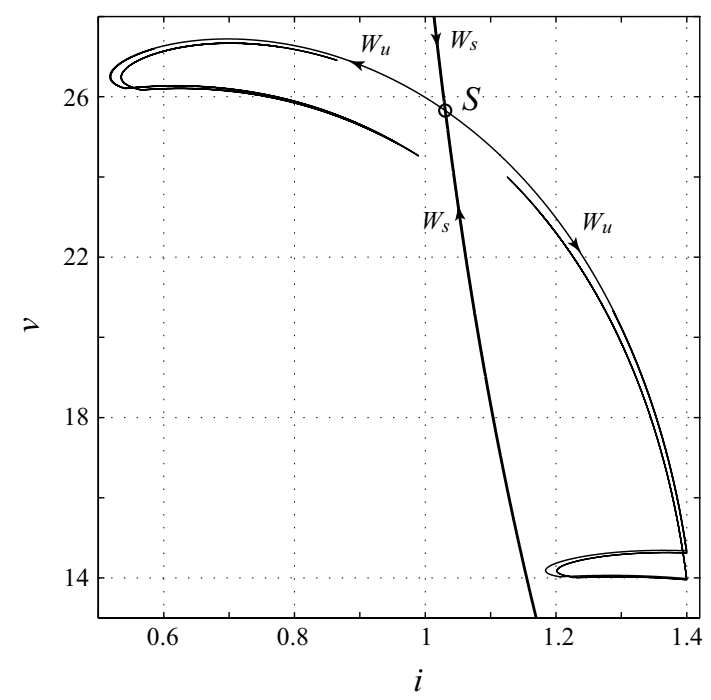

(a)

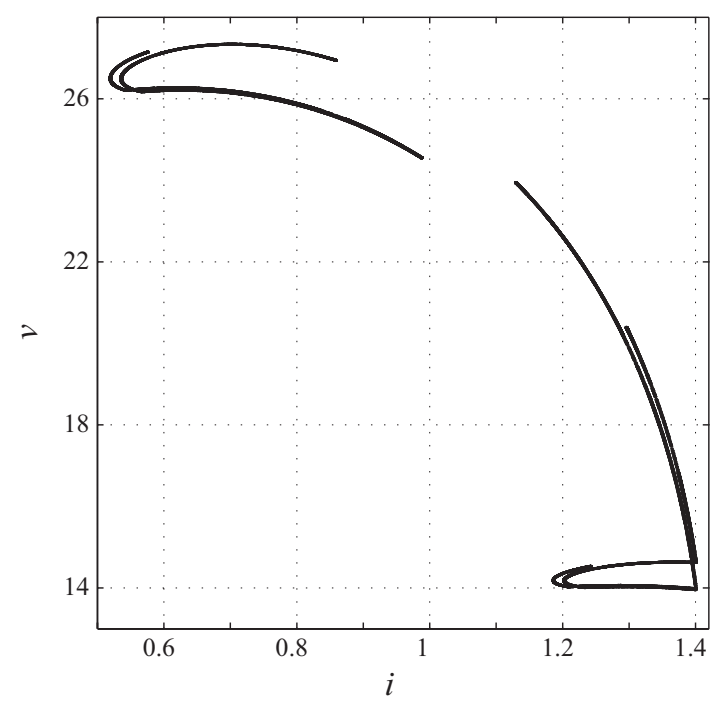

(b)

Fig. 6. (a) Stable $\left(W_{s}\right)$ and unstable $\left(W_{u}\right)$ manifolds of the saddle point $S$; (b) corresponding chaotic attractor $\left(I_{\text {ref }}=1.4 \mathrm{~A}\right)$.

map. Fig. 5 (a) shows the stable and unstable manifolds of the saddle point $S$ corresponding to the Poincaré map $p$. Here, an homoclinic intersection can be easily observed, which implies chaos. Fig. 5 (b) shows the corresponding chaotic attractor.

It has been shown previously that the current-mode controlled boost converter exhibits typical period-doubling bifurcation as $I_{\text {ref }}$ increases [6], [7]. The various stages along the chaos route can be described as follows.

1) Beyond the first period-doubling point, the fundamental fixed point becomes an unstable saddle point, which continues to exist even when the boost converter becomes chaotic. As a result, the geometrical behavior of the invariant manifolds of this saddle point will determine the global properties of the chaos being exhibited by the system.

For convenience of illustration of the effects of the invariant manifolds of the saddle point on the global properties of the boost converter, we choose the parameters as follows: $T=$ $100 \mu \mathrm{s}, R=40 \Omega, L=1.5 \mathrm{mH}, C=4 \mu \mathrm{F}$ and $E=10 \mathrm{~V}$. 


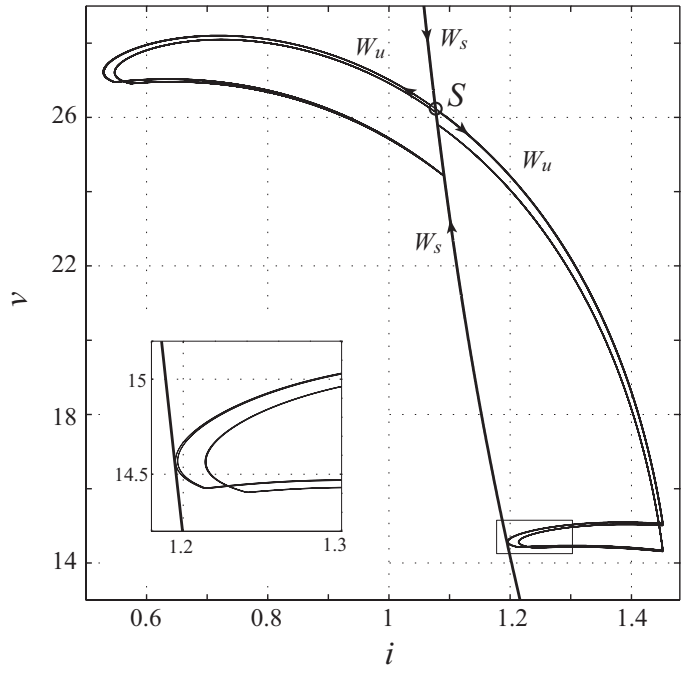

Fig. 7. The invariant manifolds at homoclinic tangency $\left(I_{\text {ref }}=1.452 \mathrm{~A}\right)$.

The invariant manifolds of the saddle point (denoted by $S$ ), when $I_{\text {ref }}=1.4 \mathrm{~A}$, are shown in Fig. 6 (a). Note that the map is non-invertible which has been rarely discussed from the horseshoe's viewpoint. From Fig. 6 (a), we see that the stable manifold does not intersect with the unstable manifold; thus no homoclinic intersection is formed. However, chaos does exist, as evidenced from the strange attractor shown in Fig. 6 (b). Note that the chaotic attractor consisting of two separate parts has been generated from a period- 2 orbit which has been resulted from the first period-doubling bifurcation. From Fig. 6, however, we may conclude that the system exhibits local chaos.

2) As $I_{\text {ref }}$ increases, the stable manifold and the unstable manifold move closer to each other. When $I_{\text {ref }}$ is around $1.452 \mathrm{~A}$, we observe a homoclinic tangency, as shown in Fig. 7.

3) Further increasing $I_{\text {ref }}$ beyond the point of homoclinic tangency, the stable and unstable manifolds intersect, giving a homoclinic connection, as shown in Fig. 8. Here, the two parts of the local chaotic attractor merge, and global chaos comes into being.

\section{CONCLUSION}

This paper studies the current-mode controlled boost converter and presents a rigorous proof for the existence of a horseshoe in the describing Poincaré map with the assistance of numerical computation. The proof is based on the result of Kennedy and Yorke [5], which provides a set of sufficient conditions for guaranteeing semiconjugation of a continuous map to the shift map, which implies chaos in the continuous map. The existence of chaos is also illustrated by observing homoclinic connections of a saddle point. For the set of parameters used, initial chaos is found to be associated with the period- 2 orbit, and is localized around two local attractors. Furthermore, the occurrence of homoclinic tangency clarifies the transition of the chaotic attractor from a local to a global one.

\section{ACKNOWLEDGMENT}

This work is supported by the Hong Kong Research Grant Council under a Competitive Earmarked Research Grant (No. PolyU 5241/03E). The authors would like to thank Prof. H. Kawakami of the University of Tokushima, for valuable discussions.

\section{REFERENCES}

[1] S. Banerjee and G. Verghese (Eds.), Nonlinear Phenomena in Power Electronics, New York: IEEE Press, 2000.

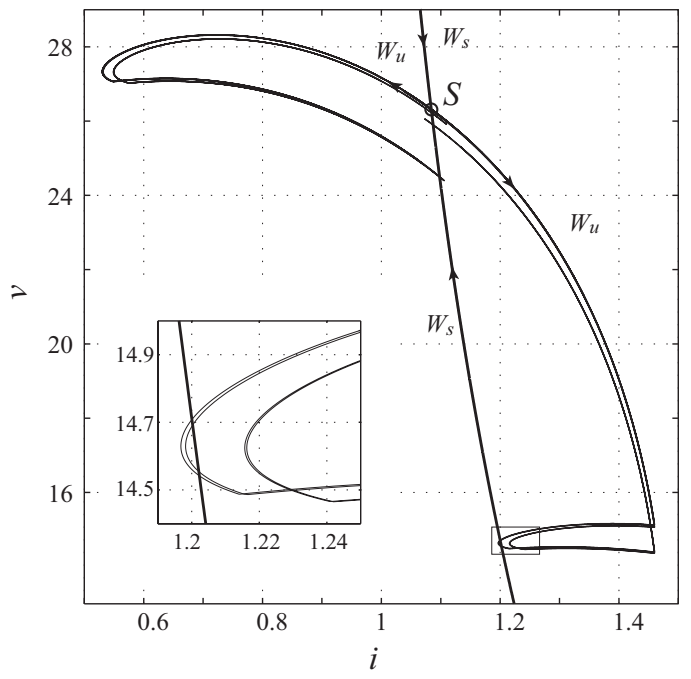

(a)

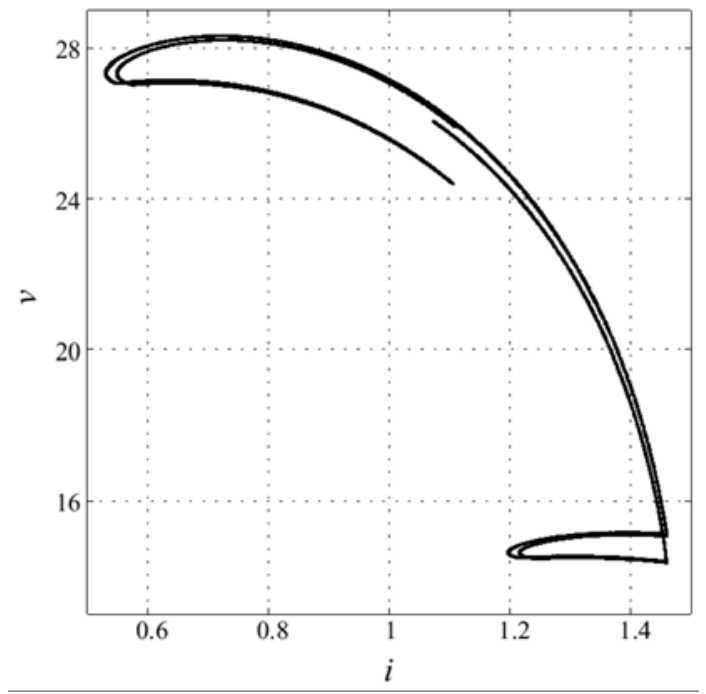

(b)

Fig. 8. (a) Homoclinic intersection of stable and unstable manifolds of the saddle point $S$; (b) global chaos due to the homoclinic intersection. ( $L_{\text {ref }}=$ $1.46 \mathrm{~A})$.

[2] C.K. Tse and M. di Bernardo, "Complex behavior of switching power converters," Proc. IEEE, vol. 90, no. 5, pp. 768-781, May 2002.

[3] C.K. Tse, Complex Behavior of Switching Power Converters, Boca Raton: CRC Press, 2003.

[4] G. Olivar, E. Fossas and C. Batlle, "Complex behavior of switching power converters," Nonlinearity, vol. 12, no. 4, pp. 1095-1121, July 2000.

[5] J. Kennedy and J.A. Yorke, "Topological horseshoes," Trans. Amer. Math. Soc., vol. 353, no. 1, pp. 2513-2530, 2001.

[6] J.H.B. Deane, "Chaos in a current-mode controlled boost dc-dc converter," IEEE Trans. Circ. Syst. I, vol. 39, no. 8, pp. 680-683, 1992.

[7] W.C.Y. Chan and C.K. Tse, "Study of bifurcation in current-programmed $\mathrm{dc} / \mathrm{dc}$ boost converters: from quasi-periodicity to period-doubling," IEEE Trans. Circ. Syst. I, vol. 44, no. 12, pp. 1129-1142, Dec. 1997.

[8] S. Wiggins, Introduction to Applied Nonlinear Dynamical Systems and Chaos, New York; Springer-Verlag, 2003.

[9] E. Ott, Chaos in Dynamical Systems, Cambridge, UK: Cambridge University Press, 2002.

[10] S. Smale, "Differentiable dynamical systems," Bull. Amer. Math. Soc., vol. 73, pp. 747-817, 1967. 